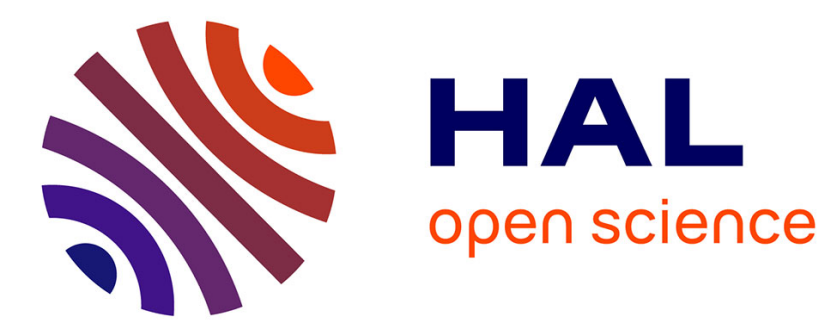

\title{
Metal mount RFID tag antenna excited by integrated loop/IC module
}

Rafael Quiroz, Jean-Marc Laheurte

\section{To cite this version:}

Rafael Quiroz, Jean-Marc Laheurte. Metal mount RFID tag antenna excited by integrated loop/IC module. Electronics Letters, 2013, 10.1049/el.2013.3092 . hal-01361746

\section{HAL Id: hal-01361746 \\ https://hal.science/hal-01361746}

Submitted on 7 Sep 2016

HAL is a multi-disciplinary open access archive for the deposit and dissemination of scientific research documents, whether they are published or not. The documents may come from teaching and research institutions in France or abroad, or from public or private research centers.
L'archive ouverte pluridisciplinaire HAL, est destinée au dépôt et à la diffusion de documents scientifiques de niveau recherche, publiés ou non, émanant des établissements d'enseignement et de recherche français ou étrangers, des laboratoires publics ou privés. 


\section{Metal mount RFID tag antenna excited by integrated loop/IC module}

\section{R. Quiroz and J.-M. Laheurte}

A simple topology is described for radio frequency identification (RFID) tags mountable on metal surfaces. The tag is built around a commercial UHF RFID module combining an IC and a coupling loop. The module is placed in the neighbourhood of a slot which is used both to couple the energy from the module to the patch-like antenna and to miniaturise the tag. A read range of almost $4 \mathrm{~m}$ is obtained in the $865-868 \mathrm{MHz}$ band with a $1.6 \mathrm{~mm}$ low-cost FR4 substrate.

Introduction: There is strong interest from many industries in tagging metal items (airplane or automotive parts, metal containers, shelves, pipes etc.) with the UHF RFID technology. The thicknesses of the published designs vary from $170 \mu \mathrm{m} \mathrm{[1]} \mathrm{to} 1 \mathrm{~cm} \mathrm{[2]} \mathrm{and} \mathrm{even} \mathrm{more.} \mathrm{For} \mathrm{the}$ tracking of large high value assets, relatively bulky tags are acceptable to guarantee the required performance and thick antennas $(>3 \mathrm{~mm})$ can protrude from the mounting surface [2]. Ultra-low-profile metal tags whose thicknesses fall into the sub-mm range (down to $200 \mu \mathrm{m}$ ) are required for flat items to achieve an aesthetic integration (e.g. the thickness of the latest Apple MacBook Air is $3 \mathrm{~mm}$ ), but only when short read ranges $(<1 \mathrm{~m})$ are acceptable $[1,3]$. Antenna thicknesses about $1-2 \mathrm{~mm}$ also find applications in numerous use cases, for instance when the tags should be curved for mounting on metallic cylinders (e.g. gas tanks and fire extinguishers) while providing medium to long read ranges (from 3 to $9 \mathrm{~m}$ ) depending on the substrate characteristics $[4,5]$.

The read range degradation is inescapable when the tags are placed above large metallic surfaces especially for small antenna thicknesses. This flows from the simultaneous drop of the antenna efficiency and detuning of its input impedance due to the high parasitic capacitance between the metallic surface and the antenna. Apart from effective multiple folded dipole [6] configurations, most solutions are based on patch type or inverted-F type designs. These antennas use shorting-pins or shorting-walls. It makes their fabrication cost higher than that of normal label-type antennas and excludes them from low-cost mass production with inexpensive materials. In this Letter, we propose a simple design for a cost-effective metal tag dedicated to medium reading distances (up to $4 \mathrm{~m}$ ) working in the European band $(865-868 \mathrm{MHz}$ ). This tag uses a module combining a loop excitation and an RFID chip. Performances are competitive with published designs of mid-range tags built on FR4 for equivalent antenna volumes and chip sensitivity, but without shorting points.

Module description: The MuTRAK module manufactured by Tagsys [7] consists of the series connection of a small loop antenna and a UHF RFID chip, the Impinj Monza 4 [8]. The measured chip read sensitivity and input impedance are $-14.7 \mathrm{dBm}$ and $1100 \Omega / / 2.11 \mathrm{pF}$, respectively, [9]. The chip impedance is $Z_{\text {chip }}=(7-\mathrm{j} 86) \Omega$ at $868 \mathrm{MHz}$. The circuit is encapsulated in a rectangular housing made of FR4 epoxy. This module must be magnetically coupled with an antenna to provide an effective radiation as the radiation resistance of the encapsulated loop is very small $(<1 \Omega)$ [10]. The $6 \mathrm{~mm}$ diameter loop is made of a $0.6 \mathrm{~mm}$ large copper microstrip. The Ansoft HFSS design of the module is shown in Fig. 1 (encapsulation is made transparent for visibility) along with its implementation inside a metallic slot.

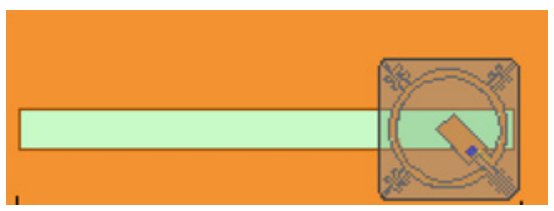

Fig. 1 MuTRAK topology and implementation showing HFSS modelling

Tag design: The antenna tag is basically an $80 \times 30 \mathrm{~mm}^{2}$ printed patch built on FR4 $\left(\varepsilon_{\mathrm{r}}=4.4, \tan \delta=0.02\right.$ and thickness $\left.1.6 \mathrm{~mm}\right)$. A $10 \times$ $2 \mathrm{~mm}^{2}$ slot is etched at the centre of one of the non-radiating edges of the patch as shown in Fig. 2. This slot extends the current path of the dominant mode on the patch, which results in the miniaturisation of the overall structure. The MuTRAK module is implemented at the slot end. The coupling mechanism is assumed to be as follows: the normal magnetic field generated by the small loop inside the module strongly excites the electrical current at the end of the slot. Then, the excited current component parallel to the patch length couples its energy with the dominant TM10 mode. To properly couple energy to the TM10 mode, it is necessary to place the module at the tip of the slot for an optimal current excitation.

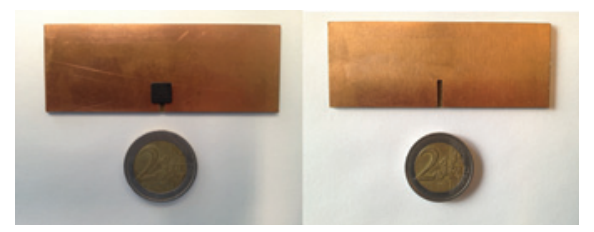

Fig. 2 Tag topology with and without MuTRAK module

The adjustment of the slot length $L_{\text {slot }}$ and slot width $W_{\text {slot }}$ is required to increase the inductance of the module loop and to match the chip capacitance. It is instructive to see in Fig. 3 the influence of the slot width on the antenna impedance. The complex conjugate of the chip impedances $\operatorname{Re}\left(Z_{\text {chip }}\right)$ and $-\operatorname{Im}\left(Z_{\text {chip }}\right)$ are also plotted for convenience. The design strategy consists of fixing the antenna resonant frequency, defined at the resistance peak, slightly above the European band $(865-868 \mathrm{MHz})$ so that the reactance curves almost cross the antenna reactance peak, whereas the antenna resistance is much lower than its peak value. For $W_{\text {slot }}$ between 4 and $5 \mathrm{~mm}$ with a resistance peak ranging from 890 to $905 \mathrm{MHz}$, the real and imaginary parts are partially matched simultaneously at $868 \mathrm{MHz}$.

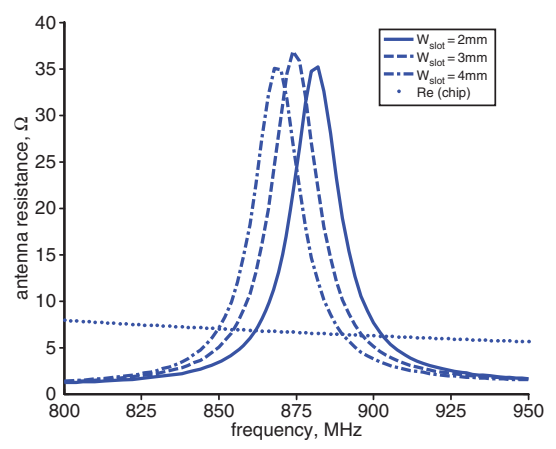

Fig. 3 Input resistance of antenna against frequency for different slot widths, $W_{\text {slot }}$

Ansoft HFSS simulations

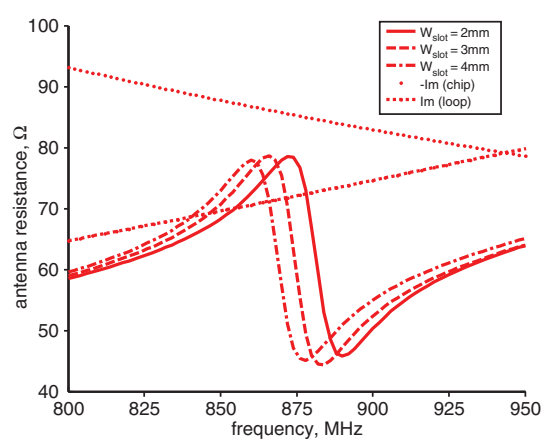

Fig. 4 Input reactance of antenna against frequency for different slot widths, $W_{\text {slot }}$

Ansoft HFSS simulations

The reactance of the module loop is also given in Fig. 4. The crosssection with the chip reactance indicates that the theoretical resonance of the module is in the upper part of the world UHF RFID band 860$960 \mathrm{MHz}$. It also shows that according to the series to parallel resonance transformation resulting from the inductive coupling [10], the antenna reactance is essentially an attenuated parallel resonance impressed on the loop response. On the other hand, the very small loop radiation 
resistance (few tenths of ohms) is strongly increased to $35 \Omega$ at resonance (Fig. 3).

The use of a commercial packaged device yields a lack of degrees of freedom and a difficulty to reach a perfect match. The design issues are limited by the fixed loop dimensions and the coupling trimming is based only on the distance between the module and the antenna. A more sophisticated matching network using a direct chip connexion [2] or a large printed coupling loop [11] allows higher coupling intensity and bandwidth. Nevertheless, the commercial strategy behind the use of the MuTRAK module, i.e. a non-contact, versatile and easy to implement integrated IC module, is powerful in many aspects.

Simulated and experimental results: The simulated gain and return loss are given in Fig. 5. Performances are: 3\% RL bandwidth $(\mathrm{RL}<-3 \mathrm{~dB})$ covering the European band, $-4 \mathrm{~dB}$ gain peak at $890 \mathrm{MHz}$ and $-5 \mathrm{~dB}$ gain peak at $868 \mathrm{MHz}$. This range of gain values is typically found with 1.5-2 mm-thick lossy FR4 substrates and label-size tags [5].

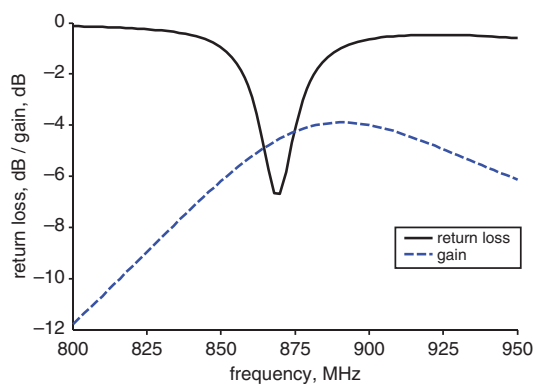

Fig. 5 Simulated return loss and gain of antenna for nominal values $\left(L=80 \mathrm{~mm}\right.$ and $\left.L_{\text {slot }}=10 \mathrm{~mm}\right)$ and for modified length $(L=70.4 \mathrm{~mm}$ and $L_{\text {slot }}=16 \mathrm{~mm}$ )

For read range measurements, the tag is placed at the centre of the $500 \times 300 \mathrm{~mm}$ metal plate. Measurements were performed with the Voyantic Tagformance System [12] as a function of frequency and are plotted in Fig. 6. The maximum read range of $3.9 \mathrm{~m}$ occurs at $870 \mathrm{MHz}$, the read range is $>3.5 \mathrm{~m}$ in the European band and $1.7 \mathrm{~m}$ in the US band (902-928 MHz).

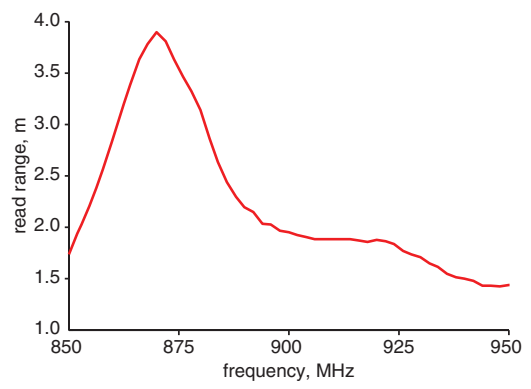

Fig. 6 Measured read range (Voyantic Tagformance)
Conclusion: A label-size metal tag built around a commercial UHF RFID module and a thin low-cost substrate has been presented. The coupling slot is used both as a mean for size reduction and as an impedance tuner. Despite inherent limitations of the commercial module used as a primary source, an optimal topology has been presented for a read range $>3.5 \mathrm{~m}$ in the European band with an Impinj Monza 4 chip.

(C) The Institution of Engineering and Technology 2013

17 September 2013

doi: 10.1049/el.2013.3092

One or more of the Figures in this Letter are available in colour online.

R. Quiroz and J.-M. Laheurte (ESYCOM, Université Paris-Est Marne-la-Vallée, 77454 Marne-la-Vallée cedex 2, France)

E-mail: laheurte@univ-mlv.fr

\section{References}

1 Rao, K., Lam, S.F., and Nikitin, P.V.: 'Wideband metal mount UHF RFID tag'. Proc. IEEE Antennas Propagation Society Int. Symp., San Diego, CA, USA, July 2008, pp. 1-4

2 Jingtian, X.I., and Terry Tao, Y.E.: 'Ultra low-profile metal tag antenna design with an emphasis on radiation efficiency'. 2013 IEEE Int. Conf. RFID, Orlando, FL, USA, April-May 2013, pp. 42-49

3 Du, G.H., Tang, T., and Deng, Y.: 'Dual-band metal skin UHF RFID tag antenna', Electron. Lett., 2013, 49, (14), pp. 858-860

4 Son, H.-W., Jeon, H.-G., and Cho, J.-H.: 'Flexible wideband UHF RFID antenna for curved metal surfaces', Electron. Lett., 2012, 48, (13), pp. 749-750

5 Mo, L., Zhang, H., and Zhou, H.: 'Broadband UHF RFID tag antenna with a pair of U slots mountable on metallic objects', Electron. Lett., 2008, 44, (20), pp. 1173-1174

6 Koo, T., Kim, D., Ryu, J., Seo, H., Yook, J., and Kim, J.: 'Design of a label-typed UHF RFID tag antenna for metallic objects', IEEE Antennas Wirel. Propag. Lett., 2011, 10, pp. 1010-1014

7 Available at http://www.tagsysrfid.com/

8 Available at http://www.impinj.com, Monza 4 RFID Tag Chips Datasheet

9 Saba, R., Deleruyelle, T., Alarcon, J., Egels, M., and Pannier, P.: 'A resistant textile tag antenna for RFID UHF frequency band'. IEEE Conf. RFID Technology and Applications, Nice, France, November 2012, pp. 203-207

10 Quiroz, R., Alves, T., Poussot, B., and Laheurte, J.-M.: 'Combined RFID tag antenna for recipients containing liquids', Electron. Lett., 2013, 49, (20), pp. 240-242

11 Kuo, S.-K., and Liao, L.-G.: 'An analytic model for impedance calculation of an RFID metal tag', IEEE Antennas Wirel. Propag. Lett., 2010, 9, pp. 603-607

12 Voyantic: 'Tagformance, product brochure' http://www.voyantic.com/ accessed September 2010 